\title{
Diagnostic accuracy of a smartphone bedside test to assess the fixation suppression of the vestibulo-ocular reflex: when nothing else matters
}

\author{
Florin Gandor ${ }^{1,2} \oplus \cdot$ Manfred Tesch $^{3} \cdot$ Hannelore Neuhauser ${ }^{4}\left(\mathbb{0} \cdot\right.$ Doreen Gruber $^{1,2} \cdot$ Hans-Jochen Heinze $^{2}$. \\ Georg Ebersbach ${ }^{1}$ (D) Thomas Lempert ${ }^{3}$ (i)
}

Received: 10 February 2020 / Revised: 20 May 2020 / Accepted: 22 May 2020 / Published online: 1 June 2020

(c) The Author(s) 2020, corrected publication 2020

\begin{abstract}
Objective Validation of a bedside test to objectify the fixation suppression of the vestibulo-ocular reflex (FS-VOR) in patients with a cerebellar syndrome and healthy controls.

Methods The vestibulo-ocular reflex and its fixation suppression were assessed by video-nystagmography (VNG) in 20

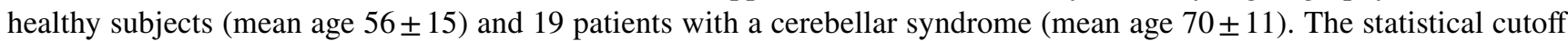
delineating normal from pathological FS-VOR was determined at the 2.5th percentile of the normal distribution of the healthy cohort. VNG was then compared to a bedside test, where eye movements were recorded with a smartphone while patients were rotated on a swivel chair at a defined speed and amplitude. These videos were rated as normal or pathological FS-VOR by six blinded raters, and results compared to VNG.

Results VNG in healthy controls showed FS-VOR with a reduction of nystagmus beats by $95.0 \% \pm 7.2($ mean \pm SD). The statistical cutoff was set at $80.6 \%$. Cerebellar patients reduced nystagmus beats by only $26.3 \% \pm 25.1$. Inter-rater agreement of the smartphone video ratings was $85 \%$. The sensitivity of the video ratings to detect an impaired FS-VOR was $99 \%$, its specificity $92 \%$. Inter-test agreement was $91 \%$.

Conclusion The smartphone bedside test is an easily performed, reliable, sensitive, specific, and inexpensive alternative for assessing FS-VOR.
\end{abstract}

Keywords Vestibulo-ocular reflex · Fixation suppression of the vestibulo-ocular reflex $\cdot$ Bedside test $\cdot$ Cerebellar syndrome

Electronic supplementary material The online version of this article (https://doi.org/10.1007/s00415-020-09947-5) contains supplementary material, which is available to authorized users.

Florin Gandor

gandor@kliniken-beelitz.de

Manfred Tesch

manfred.tesch@schlosspark-klinik.de

Hannelore Neuhauser

neuhauserh@rki.de

Doreen Gruber

gruber@kliniken-beelitz.de

Hans-Jochen Heinze

hans-jochen.heinze@med.ovgu.de

Georg Ebersbach

ebersbach@kliniken-beelitz.de

\section{Introduction}

Fixation suppression of the vestibulo-ocular reflex (FSVOR) is a reliable clinical test of cerebellar function [1, 2], and its disturbance indicative of cerebellar pathology [1,

\section{Thomas Lempert}

thomas.lempert@schlosspark-klinik.de

1 Movement Disorders Hospital, Kliniken Beelitz GmbH, Strasse nach Fichtenwalde 16, 14547 Beelitz-Heilstätten, Germany

2 Department of Neurology, Otto-Von-Guericke University, Magdeburg, Germany

3 Department of Neurology, Schlosspark-Klinik Berlin, Berlin, Germany

4 Robert-Koch-Institut, Berlin, Germany 
$3,4]$. In movement disorders, it can serve as an additional feature to differentiate idiopathic Parkinson's disease from atypical Parkinsonian syndromes such as multiple system atrophy (MSA) [5]. The gold standard of assessing FSVOR is utilizing a video-nystagmography (VNG) system and a motor-driven rotary chair. However, not only are such devices expensive and need expertise in interpretation, but they are also time and personnel consuming. Moreover, to date no defined cutoff value exists that distinguishes between normal and pathological FS-VOR. We therefore tested the FS-VOR in healthy controls and patients with pathological FS-VOR. We then developed a bedside test that allows for fast, easy to interpret and reliable assessment of FS-VOR and enables video documentation of the results.

\section{Methods}

\section{Ethical standard}

The study was approved by the central ethics committee of the Brandenburg Medical Council (S10(a)/2015) and has therefore been performed in accordance with the ethical standards laid down in the 1964 Declaration of Helsinki and its later amendments and is listed in the German Trials Register (DRKS00013968). Participants provided written informed consent prior to their inclusion into the study.

\section{Participants}

Between 2015 and 2017, 20 healthy subject (14 women, age $56 \pm 15)$ and 20 patients with cerebellar disorders (10 women, age $70 \pm 11$ years, 12 probable MSA, 3 cerebellar ischemia, 1 episodic cerebellar ataxia, 1 spinocerebellar ataxia, 1 paraneoplastic cerebellar degeneration, and 2 sporadic cerebellar degeneration) were included in the study after providing written informed consent. One screened patient with probable MSA did not show pathological FSVOR or other cerebellar pathology, was diagnosed with MSA of the Parkinsonian variant and excluded. Both groups underwent VNG and smartphone bedside testing.

\section{Video-nystagmography}

VNG was performed as described previously [6] and adapted as follows: patients were seated on a swivel chair and rotated (duration $30 \mathrm{~s}$ amplitude $90^{\circ}$, frequency $0.83 \mathrm{~Hz}$ ). A $90^{\circ}$ angle was marked on the floor, and patients were rotated by hand from side to side at a peak to peak frequency of $50 \mathrm{bpm}$ with the aid of a metronome, thereby achieving a peak velocity $118^{\circ} / \mathrm{s}$. The horizontal ocular movements were recorded using a head-mounted VNG mask (VO25, Interacoustics, Denmark). For VOR recording, the mask was shut to disable visual fixation during rotation. For FS-VOR assessment, the mask was opened and the subject asked to fixate an extended thumb of their outstretched arm during rotation. Nystagmus beats, horizontal eye position and maximum horizontal slow phase velocity (SPV) were recorded.

\section{Smartphone bedside test}

For the smartphone bedside test, patients were seated on a swivel chair and rotated in an identical way (vid.1). Patients were asked to hold a smartphone (iPhone 6S, Apple Inc., CA, USA) at arm's length and to fixate the lens. Eye movements were recorded for $30 \mathrm{~s}$ during rotation.

\section{Video rating}

FS-VOR videos of the bedside test were assessed by two medical nurses, two neurology residents, and two consultant neurologists and movement disorders specialists, all blinded to the condition of the participants. The raters dichotomously rated the videos as either pathological or normal. The latter required complete or near-complete nystagmus suppression during FS-VOR testing.

\section{Data analysis}

Data were analyzed utilizing Real Statistics Resource Pack software version 5.9.2. Distribution was calculated applying the Shapiro-Wilk test, and variance using the $F$ test. Normally distributed values of homogenous variance were compared using the non-paired parametric $t$ test, and not normally distributed values using the Mann-Whitney $U$ test.

Fixation suppression ability was quantified as percentage of the reduction of nystagmus beats or nystagmus slow phase velocity from VOR testing to FS-VOR testing. Negative values were set to zero.

Results were compared to the video ratings. Inter-test reliability between VNG and video analysis was expressed by Cohen's kappa and inter-rater reliability by Fleiss' kappa. Sensitivity and specificity were calculated. Rater-specific ROC analyses were performed and AUC values calculated.

\section{Results}

\section{Video-nystagmography and cutoff value for FS-VOR}

During VNG, VOR could be provoked both in healthy controls and patients with cerebellar pathology. Cerebellar patients showed more nystagmus beats and faster maximum SPV (Table 1). When performing FS-VOR during VNG, healthy controls were able to nearly completely suppress the VOR by mean \pm SD [range] $95.0 \% \pm 7.2[71.1-100 \%]$ 
Table 1 Absolute values and percentage reduction of (A) nystagmus counts and (B) maximum SPV during VOR and FS-VOR assessment in healthy controls and patients with a cerebellar syndrome. Values are indicated as mean $\pm \mathrm{SD}$

\begin{tabular}{lllll}
\hline & VOR & FS-VOR & \% Red & $p$ values \\
\hline (A) Nystagmus beats & & & \\
Healthy controls $(n=20)$ & $47.3 \pm 19.8$ & $2.5 \pm 3.6$ & $95.0 \pm 7.2$ & $2.6 \times 10^{-8}$ \\
Cerebellar syndrome $(n=19)$ & $63.5 \pm 21.5$ & $47.1 \pm 22.7$ & $26.1 \pm 25.1$ & 0.02 \\
$p$ values & 0.006 & $4.8 \times 10^{-8}$ & $9.7 \times 10^{-8}$ & \\
(B) Maximum SPV & & & \\
Healthy controls $(n=20)$ & $38.5 \pm 12.2$ & $2.7 \pm 3.3$ & $93.0 \pm 8.1$ & $3.1 \times 10^{-8}$ \\
Cerebellar syndrome $(n=19)$ & $50.3 \pm 9.5$ & $31.3 \pm 20.3$ & $42.4 \pm 27.6$ & 0.0005 \\
$p$ values & 0.0007 & $1.2 \times 10^{-7}$ & $2.7 \times 10^{-7}$ & \\
\hline
\end{tabular}

( $p<0.0001$; Fig. 1; example Fig. 2). Cerebellar patients were less capable of reducing nystagmus beats when performing FS-VOR and achieved a reduction of only $26.3 \% \pm 25.1$ $[0-74.7 \%](p=0.02)$, indicative of FS-VOR failure in comparison to healthy controls ( $p<0.0001$, Table 1A; Fig. 1; example Fig. 2). When assessing the maximum SPV, a nystagmus beat reduction of $93.0 \% \pm 8.1$ was calculated for healthy controls in contrast to $42.4 \pm 27.6(p<0.0001)$ in cerebellar patients (Table 1B). Moreover, cerebellar patients had significantly more nystagmus beats and higher maximum slow phase velocities than healthy controls (Table 1, example Fig. 2).

The cutoff value separating normal and pathological FSVOR was determined as $-2 \mathrm{SD}$ of nystagmus beat reduction, equivalent to the 2.5 th percentile of the normal distribution of our healthy cohort and set at $80.6 \%$ (Fig. 1, dotted line).

\section{Bedside test and video ratings}

Six raters rated the bedside test videos of the 20 healthy controls (120 ratings) and the 19 cerebellar patients (114 ratings) (video1). 113/114 (99.1\%) of the pathological and $110 / 120(91.7 \%)$ of the normal FS-VOR videos were identified correctly. Video raters achieved an inter-rater reliability of $k=0.85$ [0.76-0.93] (Fleiss' kappa [95\% CI]). The sensitivity of the video ratings to detect an impaired FS-VOR was $99 \%$ and its specificity $92 \%$.

When comparing the video ratings with the VNG results, inter-test agreement was $k=0.91$ [0.84-0.96] (Cohen's kappa [95\% CI]). Rater-specific ROC analyses revealed AUC values ranging from 0.934 to 0.997 .

\section{Discussion}

Video-nystagmography revealed that patients with a cerebellar syndrome showed significantly increased nystagmus beats and slow phase velocities during VOR testing in comparison to healthy controls. These results are in line with previous findings [7] and indicative of a release of vestibular nuclei activity from cerebellar inhibition due to cerebellar pathology [8, 9]. However, a defined cutoff value has to our knowledge not yet been defined, and we for the first time report a distribution-based threshold delineating normal from pathological fixation suppression of the vestibulo-ocular reflex (FS-VOR): during sinusoidal rotation, healthy controls were able to reduce nystagmus beats during FS-VOR by $95.0 \% \pm 7.2(p<0.0001)$. In contrast, cerebellar patients largely failed to suppress the VOR during fixation, with a nystagmus beat reduction of only $26.3 \% \pm 25.1(p=0.02)$.
Fig. 1 Percentage of nystagmus beats reduction during FS-VOR in healthy controls and patients with cerebellar pathology. Healthy controls $97.9 \%$ [93.2-100.0], cerebellar pathology 23.4\% [0.0-44.8] (median [IQR]; $* p<0.0001)$. The dotted line represents the cutoff level at $80.6 \%$ reduction of nystagmus beats, corresponding to the mean -2 SD or the 2.5 th percentile of the normal distribution of the healthy cohort

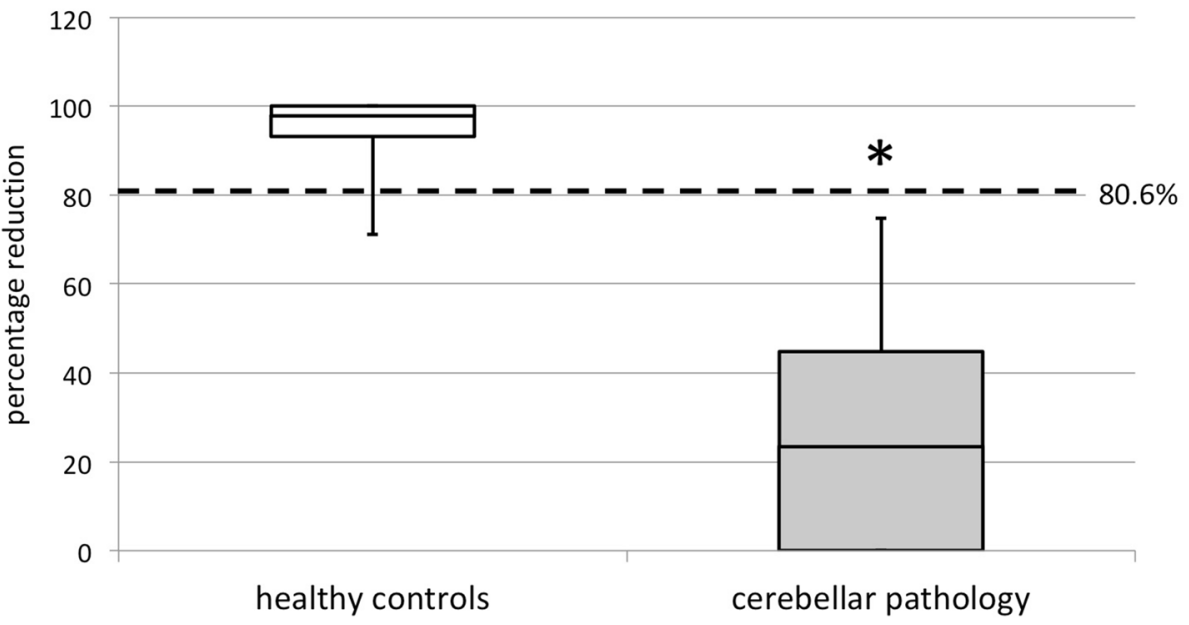


Fig. 2 Video-nystagmography of FS-VOR in one healthy subject (a) and one patient with cerebellar pathology (b), assessing the horizontal eye position (1) and horizontal slow phase velocity (SPV) (2)
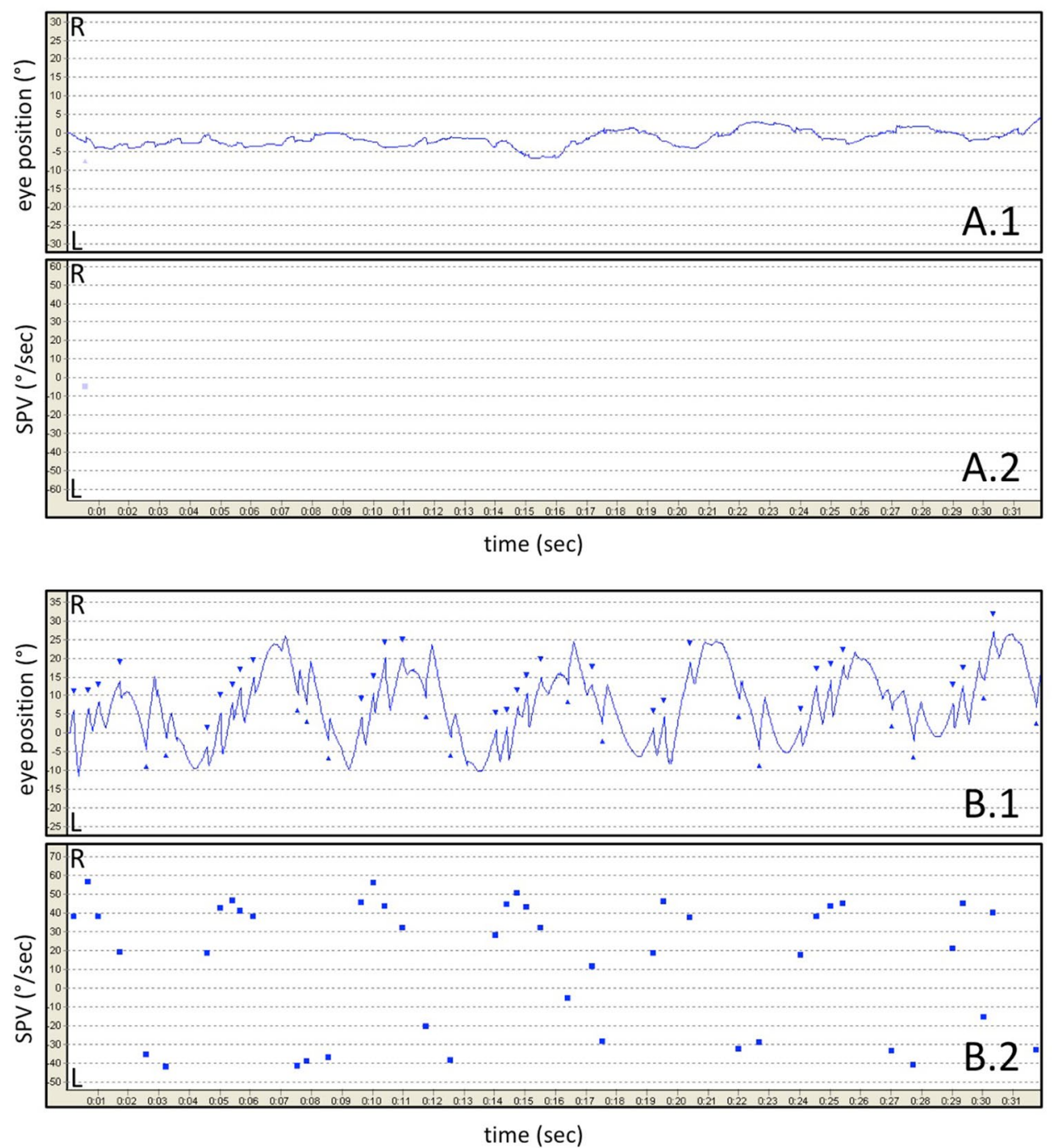

The cutoff value dividing normal from pathological FS-VOR was set at $80.6 \%$ reduction of nystagmus beats, corresponding to -2 SD from the mean or the 2.5 th percentile of the normal distribution of our healthy cohort.

The blinded video ratings of the smartphone bedside test showed an inter-rater reliability of $k=0.85$ [0.76-0.93], expressing high accordance among raters, regardless of their medical degree and qualification. Furthermore, there was an excellent inter-test reliability with $k=0.91$ [0.84-0.96]. The sensitivity of the video ratings of $99 \%$ and the specificity of 92\% suggest that this bedside test is a useful tool to detect an impaired FS-VOR.

Our proposed test has obvious advantages when compared with VNG: (1) today almost everyone owns a smartphone to perform the test, (2) swivel chairs are available everywhere, (3) the test is easily performed and (4) has a high sensitivity and specificity.

Undoubtedly, the sample size is small, and further studies in a larger cohort should verify these findings.
While performing the test, the song "Nothing Else Matters" (by Metallica, composed by James Hetfield and Lars Ulrich) can be used as a mnemonic for two purposes: firstly, the patient is advised to fixate the smartphone's lens to maintain fixation, hence "Nothing Else Matters". Secondly, the song is recorded at $50 \mathrm{bpm}$, the speed at which the $90^{\circ}$ chair rotation must be performed to achieve the peak velocity of $118^{\circ} / \mathrm{s}$.

Acknowledgements The authors thank Nadine Schmidtke and Nadine Stenzel (Schlosspark-Klinik Berlin, Germany) for performing the VNG and bedside tests, and Dr. Johanna Hemmen, RN Rowena Karl, RN Anja Kamrad, and Dr. Josephiene Schmidt (Hospital for Movement Disorders and Parkinson's disease, Beelitz-Heilstätten, Germany) for video ratings.

Author contributions FG: study design and conceptualization, data and statistical analysis, manuscript draft for intellectual content, approved the final version of this manuscript. MT: study design and conceptualization, data acquisition, manuscript revision for intellectual content, approved the final version of this manuscript. HN: data and statistical 
analysis, manuscript revision for intellectual content, approved the final version of this manuscript. DG: data and statistical analysis, manuscript revision for intellectual content, approved the final version of this manuscript. H-JH: manuscript revision for intellectual content, approved the final version of this manuscript. GE: study design and conceptualization, manuscript revision for intellectual content, approved the final version of this manuscript. TL: study design and conceptualization, manuscript revision for intellectual content, approved the final version of this manuscript.

Funding The study was supported by Neuro+e.V., Förderverein für neurologische Forschung, Heubnerweg 2, 14059 Berlin, Germany covering the costs oft he smartphone. Neuro+e.V. is a non-profit organization supporting clinical research in neurology.

Data availability Anonymized data will be shared by request from any qualified investigator.

\section{Compliance with ethical standards}

Conflicts of interest None of the authors report any financial disclosures or conflicts of interest relevant to this manuscript.

Ethical standards The study was approved by the central ethics committee of the Brandenburg Medical Council (S10(a)/2015), has therefore been performed in accordance with the ethical standards laid down in the 1964 Declaration of Helsinki and its later amendments and is listed in the German Trials Register (DRKS00013968).

Consent to participate Participants provided written informed consent prior to their inclusion into the study. The ICF is submitted with this manuscript.

Consent for publication Written informed consent was obtained from both participants recognizable on the video and submitted as supplemental material for the editors.

Open Access This article is licensed under a Creative Commons Attribution 4.0 International License (https://creativecommons.org/licenses/ by/4.0/), which permits use, sharing, adaptation, distribution and reproduction in any medium or format, as long as you give appropriate credit to the original author(s) and the source, provide a link to the Creative Commons licence, and indicate if changes were made. The images or other third party material in this article are included in the article's Creative Commons licence, unless indicated otherwise in a credit line to the material. If material is not included in the article's Creative Commons licence and your intended use is not permitted by statutory regulation or exceeds the permitted use, you will need to obtain permission directly from the copyright holder. To view a copy of this licence, visit http://creativecommons.org/licenses/by/4.0/.

\section{References}

1. Dichgans J, von Reutern GM, Römmelt U (1978) Impaired suppression of vestibular nystagmus by fixation in cerebellar and noncerebellar patients. Arch Psychiatr Nervenkr 226:183-199. https ://doi.org/10.1007/BF00341710

2. Halmagyi GM, Gresty MA (1979) Clinical signs of visual-vestibular interaction. J Neurol Neurosurg Psychiatry 42:934-939. https ://doi.org/10.1136/jnnp.42.10.934

3. Gauthier GM, Vercher JL (1990) Visual vestibular interaction: vestibulo-ocular reflex suppression with head-fixed target fixation. Exp Brain Res 81:150-160. https://doi.org/10.1007/BF00230111

4. Strupp M, Kremmyda O, Adamczyk C et al (2014) Central ocular motor disorders, including gaze palsy and nystagmus. J Neurol 261:542-558. https://doi.org/10.1007/s00415-014-7385-9

5. Anderson T, Luxon L, Quinn N et al (2008) Oculomotor function in multiple system atrophy: clinical and laboratory features in 30 patients. Mov Disord 23:977-984. https://doi.org/10.1002/ mds.21999

6. Blödow A, Heinze M, Bloching MB et al (2014) Caloric stimulation and video-head impulse testing in Ménière's disease and vestibular migraine. Acta Otolaryngol 134:1239-1244. https:// doi.org/10.3109/00016489.2014.939300

7. Shaikh AG, Marti S, Tarnutzer AA et al (2011) Ataxia telangiectasia: a "disease model" to understand the cerebellar control of vestibular reflexes. J Neurophysiol 105:3034-3041. https://doi. org/10.1152/jn.00721.2010

8. Walker MF, Zee DS (2005) Cerebellar disease alters the axis of the high-acceleration vestibuloocular reflex. J Neurophysiol 94:3417-3429. https://doi.org/10.1152/jn.00375.2005

9. Walker MF, Zee DS (1999) Directional abnormalities of vestibular and optokinetic responses in cerebellar disease. Ann N Y Acad Sci 871:205-20. https://www.ncbi.nlm.nih.gov/pubmed/10372 073. Accessed 25 Feb 2019 